\title{
Comparison of the Effects of Electrolytic and Chemical Destruction of the Red Nucleus on the Compensatory Capacity of Rats with Rubrospinal Tract Lesions
}

\author{
V.V. Fanardjian, ${ }^{\dagger}$ E.V. Papoyan, V.I. Pogossian, and O.V. Gevorkyan \\ L.A. Orblei Institute of Physiology, National Academy of Sciences of Armenia, \\ 22 Orblei Bros. Str., 375028, Yerevan, Republic of Armenia
}

SUMMARY

Transection of the rubrospinal tract in rats, performed before lesion of the red nucleus, resulted in the facilitated recovery of motor activity and operantly conditioned reflexes. Such facilitation was absent when the red nucleus is lesioned alone. This phenomenon is explained by the switching of descending influences on the corticospinal tract through the participation of the following system: red nucleus - inferior olive - cerebellum - ventrolateral thalamic nucleus-cerebral cortex. The above mentioned facilitating influence on the recovery process was particularly prominent in rats with quinolinic acid-induced lesion of the red nucleus. Under these conditions, the cerebellar ascending fibers to the ventrolateral thalamic nucleus were preserved. Decreased facilitated recovery following electrolytic lesion of the red nucleus suggests the existence of additional cerebello-cortical pathways for the realization of the switching phenomenon.

\section{KEYWORDS}

Plasticity, operantly conditioned, switching of descending influences

\footnotetext{
Corresponding author:

tel: +(3742) 27-04-41; fax: +(3742) 27-22-47

e-mail:vvfanar@sci.am
}

\section{INTRODUCTION}

Transection of the rubrospinal tract (RST) before lesion of the red nucleus ( $R N)$ has been shown to facilitate the recovery of operantly conditioned reflexes and the compensatoryrehabilitation process in rats. $\mathrm{RN}$ lesion alone, however, not preceded by transection of RST, leads to prolonged disturbances of motor activity and operantly conditioned reflexes (Kennedy, 1990). On this basis, it has been proposed that the quick recovery of motor activity after the transection of RST is a consequence of activation of the rubro-olivary projection (Rossi \& Strata, 1997), leading to switching of motor activity under control of the corticospinal tract (Kennedy \& Humphrey, 1987). This switching is realized by the participation of the cerebellum and the ventrolateral thalamic nucleus (VL), which transmit the information to the cerebral cortex. During this process, VL functions as a basic relay center for the ascending cerebellar influences on the cerebral cortex, leading to the switching of cortical control for the new context of motion (Kennedy \& Humphrey, 1987; Kennedy, 1990). In a previous paper (Fanardjian et al., 1995), we demonstrated the role of VL in the process of descending motor switching in the operantly conditioned rat; the preliminary destruction of VL was shown that to greatly hamper the process of switching. Comparison of the effects of electrolytic and chemical lesion (by quinolinic acid) of $\mathrm{RN}$ may clarify the role of VL in the mechanisms of switching of the supraspinal influences. Electrolytic lesion of $\mathrm{RN}$ not only leads to 
damage of rubral cells, but also results in the interruption of the cerebello-thalamic fibers passing through $\mathrm{RN}$ to VL that give collaterals to rubral neurons (Toyama et al., 1970; Shinoda et al., 1988). Therefore, electrolytic lesion of $\mathrm{RN}$ leads to disturbance of activity of RST and VL systems. On the other hand, injection of quinolinic acid into $\mathrm{RN}$ destroys the somata of cells, whereas the cerebello-thalamic fibers are preserved (Kennedy \& Humphrey, 1987). We have addressed the role of the cerebello-thalamic system in the switching mechanism of descending motor systems. The results of the two experiments mentioned above on operantly conditioned rats are presented below.

\section{METHODS}

Predominantly male albino rats $(n=42), 2$ to 3 months old and weighing 210 to $250 \mathrm{~g}$, were trained to move and balance on a slowly rotating horizontal bar ( 9 rotations per minute), which was $30 \mathrm{~cm}$ in length and $3 \mathrm{~cm}$ in diameter. The horizontal bar was fixed at a height of $90 \mathrm{~cm}$ above a soft cushion. Conditioning for the instrumental reflex of equilibrium was determined as the time spent on the rotating bar. The maximum time of each trial was $5 \mathrm{~min}$. The trial was repeated 10 times per session, with a 1-min interval between each trial. The criterion for conditioned performance was the rat's ability to balance on the rotating bar for no less than $250 \mathrm{~s}$ (Kennedy \& Humphrey, 1987).

After conditioning for stable instrumental reflexes, rats were anesthetized intraperitoneally using sodium pentobarbital (Nembutal, Tallinn) (40 mg/kg body weight), and then the surgical procedures were performed. After several sequential experimental procedures, the vast majority of rats survived and underwent further experiments, which included two basic groups with electrolytic and chemical destruction. Each group underwent the following experimental procedures:
1. operant conditioning;

2. isolated electrolytic and chemical destruction of $\mathrm{RN}$;

3 . preliminary transection of the spinal cord dorsolateral funiculus (DLF) and further electrolytic and chemical destruction of the red nucleus;

4. injection of horseradish peroxidase (HRP) into $\mathrm{VL}$ of rats with chemically lesioned $\mathrm{RN}$.

In 21 rats, the DLF (which contains RST) was transected unilaterally at the upper cervical spinal cord (C 3). Contralateral to the above mentioned transection, $\mathrm{RN}$ was lesioned electrolytically or chemically, guided by the stereotaxic coordinates (Paxinos \& Watson, 1986). In 11 animals, RN alone was lesioned. Thus, after the experimental procedure, 32 rats were taken for further analysis. After each procedure, reconditioning of instrumental reflexes was performed, and a general picture of the changes in dynamic behavior was constructed.

Electrolytic lesion of $\mathrm{RN}$ was effected by passing direct anodic current $(2.5 \mathrm{~mA})$ for 10 to $15 \mathrm{~s}$ through a steel unipolar electrode, which was insulated except for the tip $(100 \mu \mathrm{m})$ (14 rats). The neurons of $\mathrm{RN}$ were destroyed chemically by injecting a solution of $2 \%$ quinolinic acid (8 rats). The solution was prepared by sonicating $100 \mathrm{mg}$ of acid powder (Sigma) in $5 \mathrm{~mL}$ of phosphatebủffered saline $(\mathrm{pH}$ 4.0). Quinolinic acid was injected under pressure with the by a glass micropipette connected to a Hamilton syringe. The Hamilton syringe was filled with high-vacuum silicone grease and later by a solution of $2 \%$ quinolinic acid. The syringe with the micropipette was fixed to an electrode carrier. The tip of the micropipette was oriented onto $\mathrm{RN}$ according to the stereotaxic coordinates (Paxinos \& Watson, $1986)$ and 0.8 to $1.0 \mu \mathrm{L}$ of quinolinic acid was slowly injected, as determined by the meniscus of the silicone grease.

Preservation of the cerebello-thalamic fibers passing through $\mathrm{RN}$ was determined in the rats operated on by the above mentioned methods. In two control experiments, 6 to 7 days after the unilateral injection of quinolinic acid into $\mathrm{RN}$, HRP was injected into VL. For this purpose, a 
solution of 5\% HRP (Sigma, Type VI) in $0.1 \mathrm{M}$ Tris-buffered saline, $0.5 \mathrm{M} \mathrm{KCL}$ and $0.5 \mathrm{M}$ DMSO (pH 8.3) was injected into VL of two anesthetized rats. The injection was made with a glass micropipette (tip diameter $=20$ to $30 \mu \mathrm{m}$ ) that was stereotaxically guided to VL. HRP was injected microiontophoretically (positive current of 16 to $20 \mathrm{nA}, 200 \mathrm{~Hz}, 5 \mathrm{~min}$ series duration, having a 5-min interval at a four times recurrence period). During the injection, the micropipette remained for $30 \mathrm{~min}$ in the brain. Anesthesia was repeated $48 \mathrm{~h}$ later, and brain perfusion was carried out. The brain was divided into blocks from which serial sections $(75 \mu \mathrm{m})$ were made and HRP was histochemically revealed using tetramethyl benzidine (Mesulam, 1978).

In all experimental animals, neurological investigations of motor disorders were conducted in parallel with the study of instrumental reflexes. Characteristic postoperative disorders, such as impairment of the limbs, tilt of the head, and reflex of bar grasping, reflex of the limb flexion and extension to squeezing, reflex of the body and head rectification at the moment of fall, were tested. Under standard conditions of vivaria, all animals were segregated into groups of 4 to 5 rats. After the above experiments were concluded, morphological investigations of the damaged structures were conducted. Experimental animals were sacrificed with a lethal dose of sodium pentobarbital, and brains were extracted and fixed in $10 \%$ neutral formalin. Sections of the brain were stained by Nissl's method. The Student's t-test was used to determine the extent of the difference in the recovery of motor activity and instrumental reflexes (Pollard, 1977).

\section{RESULTS}

In all rats that were operantly conditioned, the defined criterion for stable reflexes was reached on the $2^{\text {nd }}$ to $4^{\text {th }}$ experimental day (mean days $2.5 \pm 0.2$; $\mathrm{SD} ; \mathrm{n}=42$ ) (Fig. 1, In). Unilateral transection of DLF

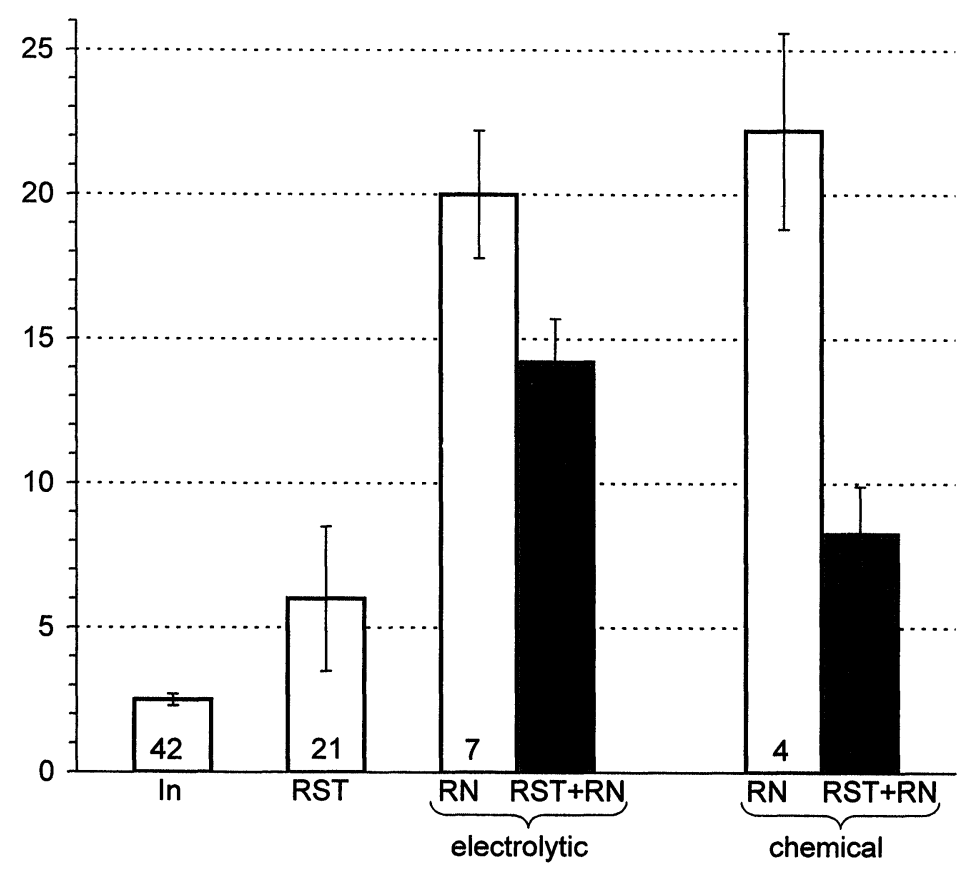

Fig. 1: Histogram comparing mean values \pm SD from the mean in the number of days required to reach criterion values of performance of instrumental reflexes (ordinate). Abscissa indicates the training of reflexes in intact rats (In), after transection of the dorsolateral funiculus (here called the rubrospinal tract, RST), electrolytic lesion red nucleus (RN) alone, transection of RST and subsequent electrolytic lesion of RN (RST+RN, electrolytic), chemical lesion of RN alone (RN), transection of RST and subsequent chemical lesion of RN (RST+RN, chemical). The numerical value within each column indicates the number of rats in this series of experiments. 
at the third cervical vertebra (C 3) was performed $(n=21)$. This procedure led to paresis of the homolateral forelimbs and hindlimbs, which subsided in a few days. Rats were taken for the experiments on the $3^{\text {rd }}$ to $7^{\text {th }}$ postoperative days. Stable instrumental reflexes were developed after 3.5 to 8.5 experimental days (mean days $6.0 \pm 2.5 ; n=21$ ) (Fig. 1, RST). Then the animals with stable instrumental reflexes underwent either electrolytic or chemical lesion of $\mathrm{RN}$.

\section{Effects of electrolytic lesion of the red nucleus}

In seven rats, electrolytic lesion of the contralateral $\mathrm{RN}$ was performed from the $16^{\text {th }}$ to $17^{\text {th }}$ day after unilateral transection of DLF. The lesion resulted in paresis of the forelimbs and hindlimbs, contralateral to the lesioned side. Motor disorders were head tilt to the same side and wide positioning of the limbs during movement. On the $7^{\text {th }}$ to the $10^{\text {th }}$ day after the second operation, the conditioning of instrumental reflexes resumed and then became stable by 12.6 to 16.0 experimental days (mean days $=14.2 \pm 1.5 ; \mathrm{n}=7$ ) (Fig. $1, \mathrm{RST}+$ RN electrolytic, and Fig. 2B).

In another series of experiments (seven rats), $\mathrm{RN}$ alone was electrolytically lesioned. The resultant motor disorders were similar to those observed in the preceding series. Motor deficit was compensated for on the $5^{\text {th }}$ to $10^{\text {th }}$ pstoperative day. It should be

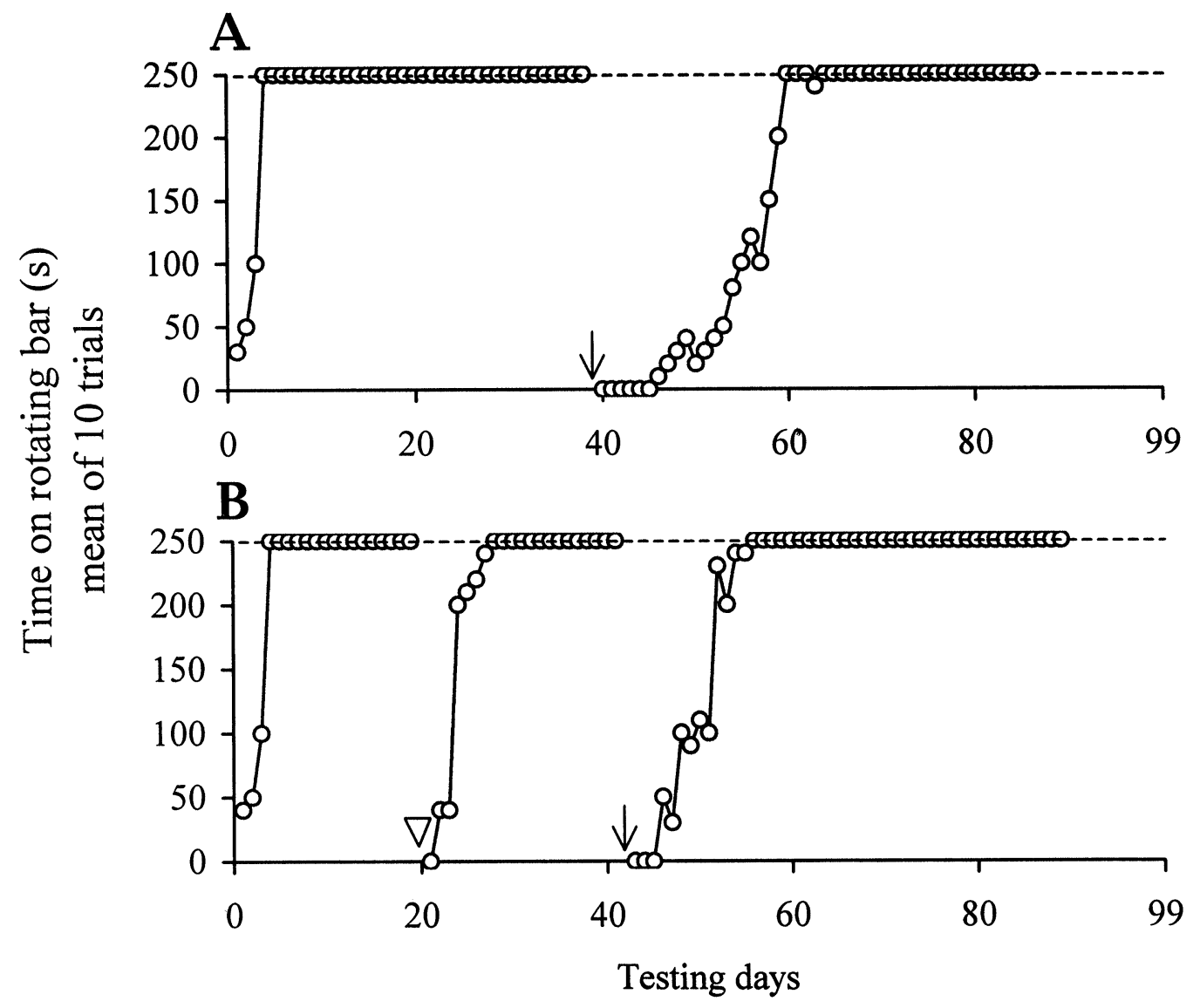

Fig. 2: Effects of transection of the dorsolateral funiculus and electrolytic lesion of the RN on the performance of learned instrumental reflexes in two rats. (A) RN alone was lesioned. (B) RN lesion was made after transection of the DLF. The progression of the reflexes is shown in days (abscissa). The mean value (in seconds) of 10 trials on the rotating bar is plotted for each training day (ordinate). The dashed line is the 250-s criterion level of performance. The open arrowhead designates the day of the DLF transection; the arrow indicates the day of RN lesion. 
noted that the intensity of motor deficit was dependent on the degree of lesion (see Fig. 3). Conditioning of instrumental reflexes resumed, reaching $100 \%$ by 17.5 to 22.2 experimental days (mean days, $20.0 \pm 2.2 ; \mathrm{n}=7$ ) (Fig. 1, RN, electrolytic, and Fig. 2A). Thus, following $\mathrm{RN}$ lesion alone, motor activity and instrumental reflexes recovered in $20 \mathrm{~d}$. In contrast, however, recovery was faster (14.2 d) in animals that underwent transection of DLF before $\mathrm{RN}$ lesion (statistically significant at $\mathrm{p}<0.005$ ).
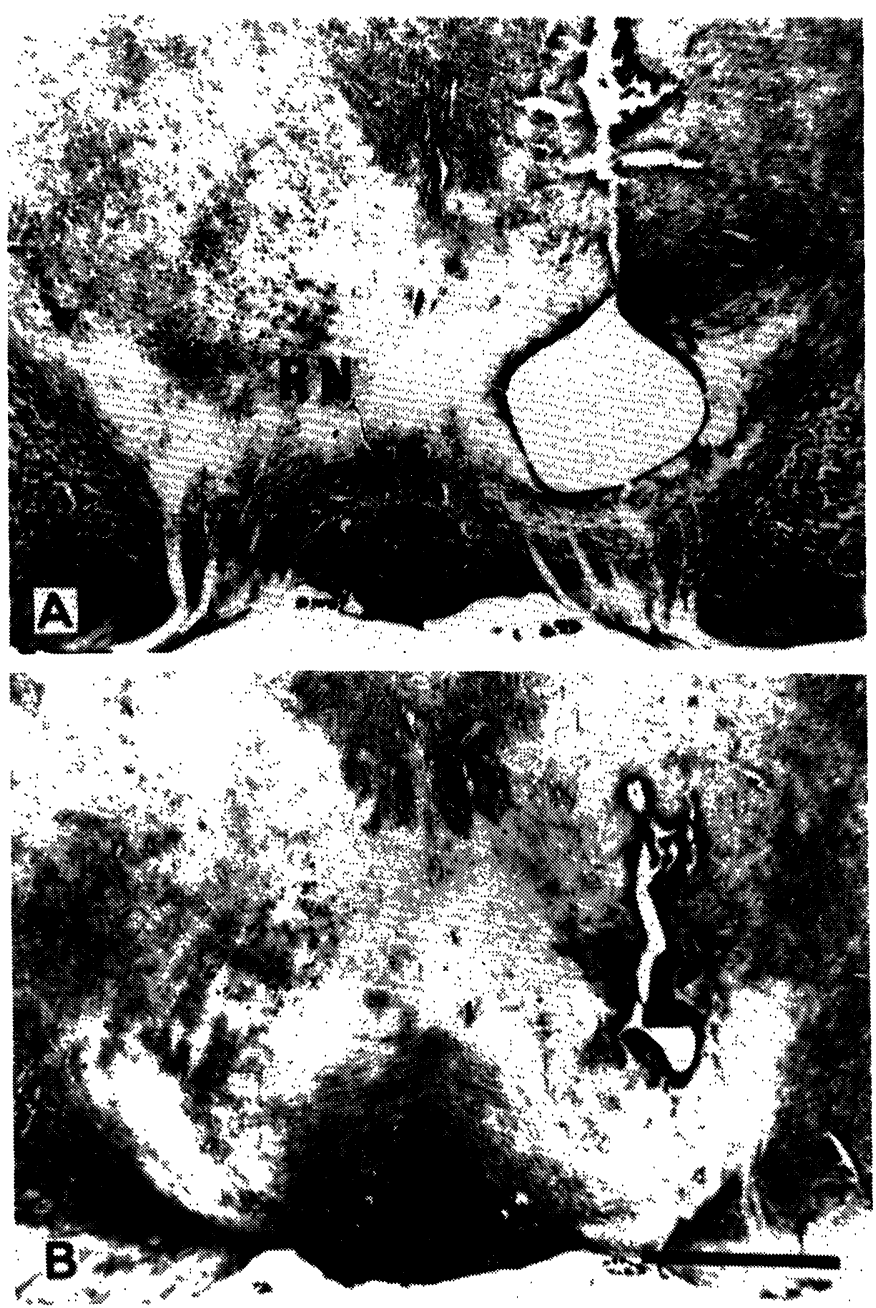

Fig. 3: Microphotographs of the frontal sections from the midbrain of rats with unilateral electrolytic completely (A) and incompletely (B) lesioned RN. (A) The cells in the $\mathrm{RN}$ are easily recognized on the unaffected side. On the opposite right side, no nerve cells are left in the area. (B) The incompletely lesioned $\mathrm{RN}$, with some unaffected nerve cells. Stained by Nissl's method. Bar $=1 \mathrm{~mm}$.

\section{Effects of chemical lesion of the red nucleus}

After compensation of motor disorders and recovery of instrumental reflexes (18.0 to 19.0 experimental days) following unilateral transection of DLF, the contralateral $\mathrm{RN}$ was chemically lesioned in four rats. After the injection of quinolinic acid into $\mathrm{RN}$ following recovery from anesthesia, tonic spasms, such as extension of the neck, back, and tail, occurred in response to pinch and to loud sound. During this period, the limbs ipsilateral to the lesion were flexed, while those contralateral were extended. This response was markedly intense in the forelimbs. Occasionally, small oscillatory movements of the head were observed. After 3 to $4 \mathrm{~h}$, when the rats had recovered from the anesthesia, these phenomena disappeared. A clinical picture characteristic of lesion of $\mathrm{RN}$ developed. A characteristic gait was observed in the animals, as they moved with their limbs apart and dragged their hindlimbs. Rats were taken for the experiments on the $7^{\text {th }}$ to $8^{\text {th }}$ day after the second operation. Stable instrumental reflexes developed by experimental days 6.0 to 10.0 (mean days 8.25 $\pm 1.65 ; \mathrm{n}=4$ ) (Fig. 1 , RST $+\mathrm{RN}$ chemical, and Fig. 4C, 4D).

In another group of four animals, the unilateral $\mathrm{RN}$ alone was chemically destroyed. Motor disorders developed that were similar to those observed in the experimental rats from the previous series of experiments. On the $6^{\text {th }}$ to $11^{\text {th }}$ postoperative day, the rats were taken for further experiments. Stable instrumental reflexes developed by 18.0 to 27.0 experimental days (mean days 22.2 $\pm 3.4 ; n=4$ ) (Fig. 1, RN, chemical, and Fig. 4A, 4B). Similar statistically significant differences $(p<0.005)$ in the times of recovery of motor activity and of instrumental reflexes were observed during the experiments involving chemical lesion of $\mathrm{RN}$ alone $(22.2 \mathrm{~d})$ and lesion of RN after transection of DLF (8.25 d).

HRP injection demonstrated that quinolinic acid destroyed the somata of rubral neurons, whereas the cerebello-thalamic fibers passing through RN were preserved. Figure 5 shows that unilateral destruction of $\mathrm{RN}$ was accomplished by 

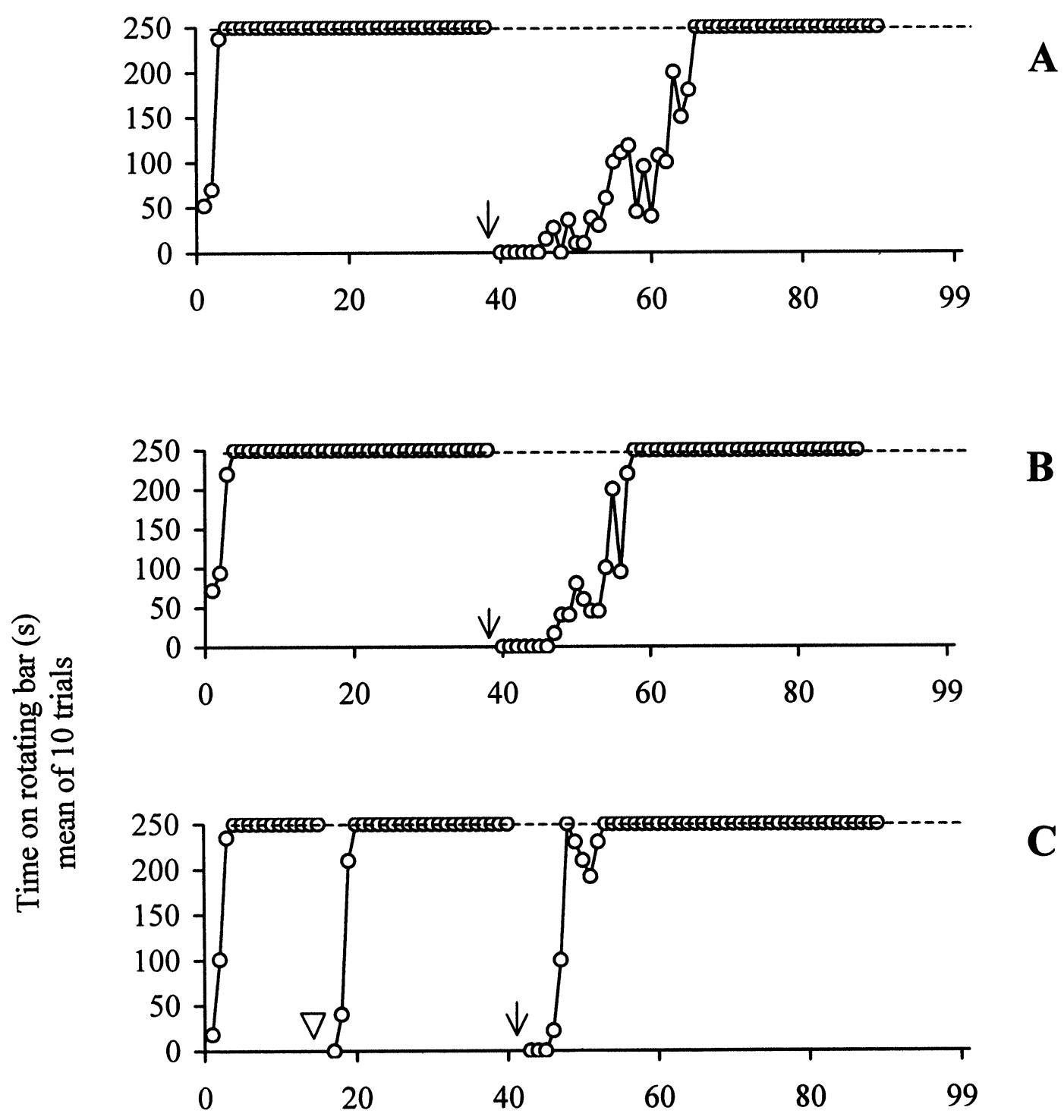

B

C

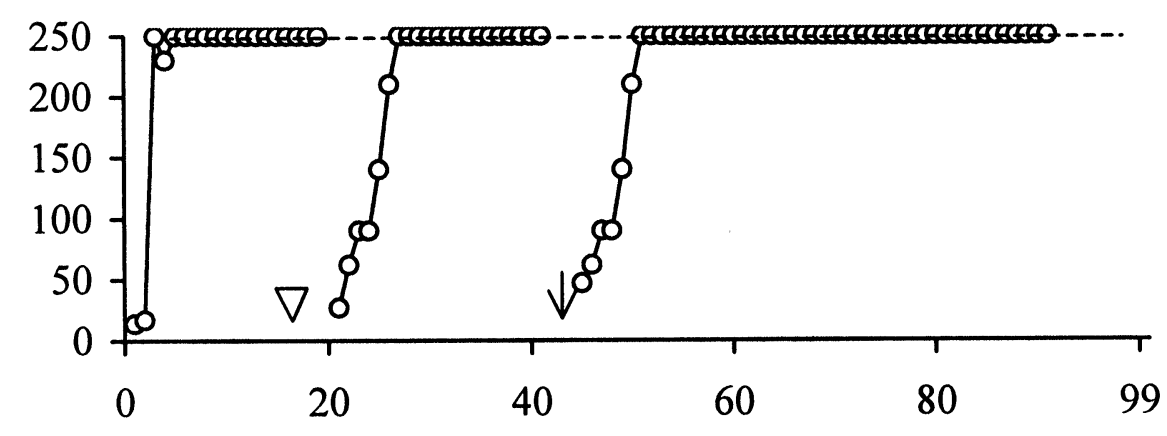

$\mathbf{D}$

Testing days

Fig. 4: Effects of transection of the dorsolateral funiculus and chemical lesion of $R N$ on performance of learned instrumental reflexes in four rats (A, B, C, D). A and B: RN alone was lesioned. C and D: RN lesion was made after transection of the DLF. The designations are the same as those in Fig. 2. 

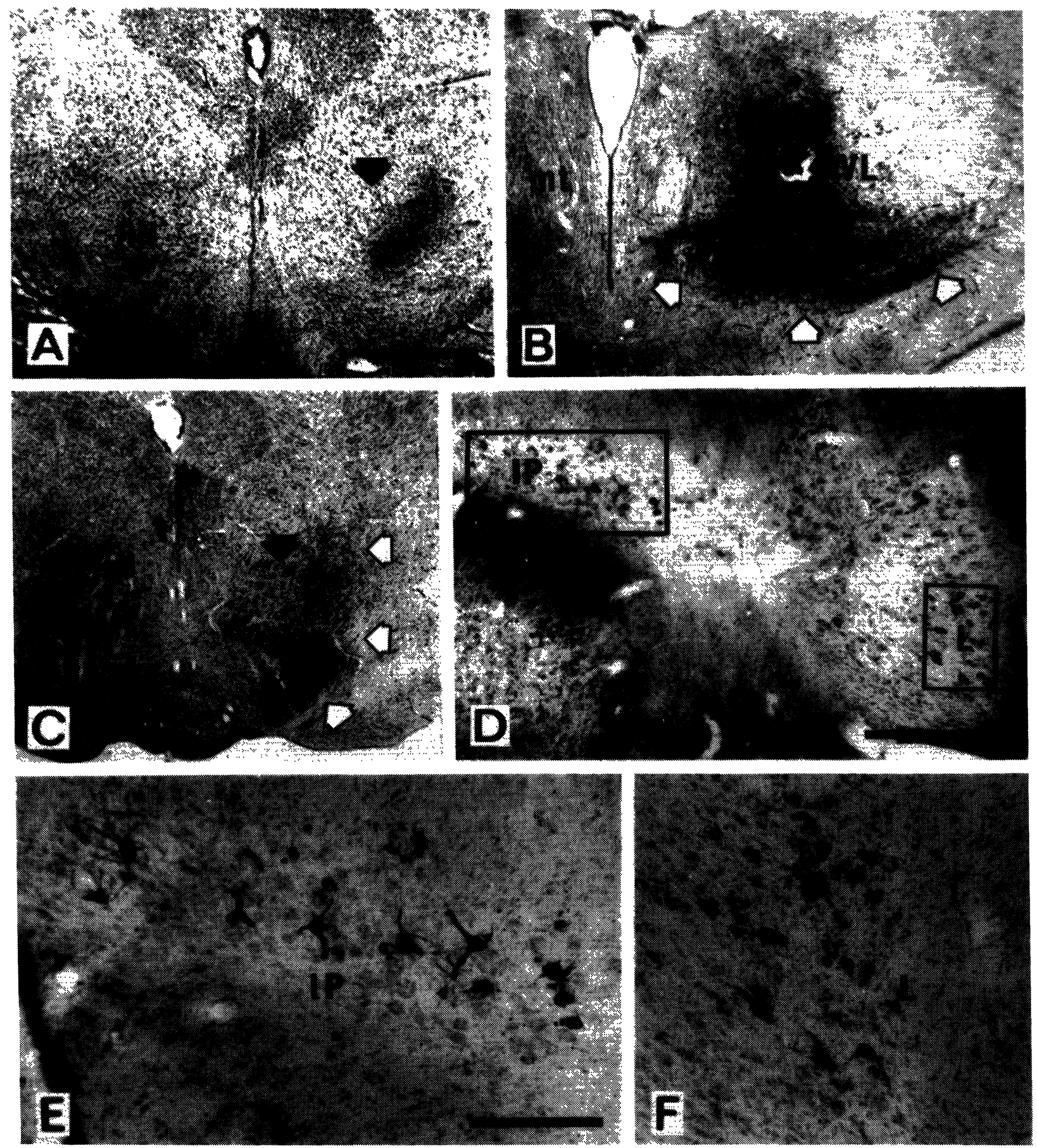

Fig. 5: Microphotographs of the frontal sections of the (A,C) rat midbrain, (B) the thalamus, and (D,E,F) cerebellar nuclei. The effects of chemical lesion of RN by injection of $(A, C)$ local quinolinic aid and $(B)$ local microiontophoretic HRP into the VL, on day 7 after chemical lesion. Microphotographs of HRP-labeled efferent neurons of nucleus interpositus (IP) and nucleus lateralis (L) of the cerebellum (D). Magnifications of the insets shown in (D) for IP and L (E and F, respectively). The closed arrowheads designate destroyed neurons of RN; the open arrowheads designate retrogradely labeled cerebello-thalamic fibers passing through RN (C). Section (A) was stained by Nissl's method; in sections (B to F), the enzyme was histochemically revealed using tetramethyl benzidine. Bar: $1 \mathrm{~mm}=500$ in A to $C, 1 \mathrm{~mm}=200$ in $D$; $\mathrm{mt}=$ mammillo-thalamic tract. 
quinolinic acid. In contrast, the opposite side of $\mathrm{RN}$ was completely unaffected $(5 \mathrm{~A}, 5 \mathrm{C})$. Labeling of neurons in the nucleus interpositus and nucleus lateralis of the cerebellum (5D), following HPR injection into VL (5B), indicated that the cerebellothalamic fibers passing through $\mathrm{RN}$ (5C) were preserved. The higher magnification $(5 \mathrm{E}, 5 \mathrm{~F})$ emphasizes this effect. Thus, the unilateral injection of HRP into VL after unilateral $\mathrm{RN}$ destruction clearly demonstrated the absence of rubrospinal and other somatic structures in the ipsilateral $\mathrm{RN}$, whereas cerebello-thalamic fibers were largely preserved.

\section{DISCUSSION}

The experiments presented here show the facilitating influence of preliminary transection of RST on the recovery of motor activity and instrumental reflexes in rats after lesion of $\mathrm{RN}$, which agrees with the finding of Kennedy and Humphry (1987). It should be noted that the facilitating influence is prominent in rats with chemically lesioned $\mathrm{RN}$ (specifically. under conditions of cerebello-thalamic fiber preservation). The facilitation time was calculated as the difference between the times of recovery after RN lesion alone and after $\mathrm{RN}$ lesion preceded by transection of RST. The respective facilitation times for electrolytic and chemical lesions were $5.8 \mathrm{~d}$ (compare 20.0 and $14.2 \mathrm{~d}$ ) and 14 days (22.2 and $8.25 \mathrm{~d})$.

As previously hypothesized, VL is a major relay nucleus for cerebellar influences on the cerebral cortex and leads to a switching of cortical control for the new context of motion. Earlier studies conducted in our laboratory (Fanardjian et a., 1995) showed that preliminary lesion of VL strongly hampers the switching of motor activity that is under the control of the corticospinal tract in rats with damaged RST and $\mathrm{RN}$. In some animals, however, unstable instrumental reflexes remained after three consecutive operations. The findings of the present study agree with those results. Our results, obtained by electrolytic and chemical lesion of $\mathrm{RN}$, confirm that the recovery of motor activity and instrumental reflexes occurs even after the interruption of the cerebello-thalamic fibers. Therefore, we predict that transmission of the ascending cerebellar influences on the cerebral cortex can be realized even through alternative cerebello-cortical pathways (see Mauk, 1997). Such pathways may involve different thalamic structures, as well as the reticular formation of the brainstem (Ito, 1984). Among the latter, the tectal structures of the midbrain, namely, the ventral tegmental region, the pars compacta of substantia nigra, and the retrorubral region, require great attention. The above mentioned structures contain a higher number of dopaminergic neurons (Dahlstrom \& Fuxe, 1964), receive direct connections from the lateral and interpositus cerebellar nuclei (Perciavalle et al., 1980) and send ascending projections to cortical structures, thus, taking part largely in motor activity (Oades \& Halliday, 1987; Piazza et al., 1987). Therefore, a comparison of the results of chemical and electrolytic lesion of $\mathrm{RN}$ confirms the presence of additional cerebello-cortical ascending pathways that might explain the switching effect. Such switching is, indeed, one of the basic compensatory mechanisms of destroyed motor function.

\section{REFERENCES}

Dahlstrom A, Fuxe K. Evidence for the existence of monoamine-containing neurons in the central nervous system. Acta Physiol. Scand 1964; 62: Suppl. 232.

Fanardjian VV, Papoyan EV, Gevorkyan OV, Pogossian VI. The influence of ventrolateral thalamic nucleus lesion on the process of switching of the descending influences on the training of instrumental reflexes in the rats. DNAN Armenia 1995; 95: 58-62 (in Russian).

Ito M. The Cerebellum and Neural Control. New York: Raven Press 1984.

Kennedy PR. Corticospinal, rubrospinal and rubroolivary projections: a unifying hypothesis. Trends Neurosci 1990; 13: 474-479.

Kennedy PR, Humphry DR. The compensatory role of the parvocellular division of the red nucleus in operantly conditioned rats. Neurosci Res 1987; 5: 39: 39-62. 
Mauk MD. Role of cerebellar cortex and nuclei in motor learning: Contradictions or Clues? Neuron 1997; 18 : 343-346.

Mesulam MM. Tetramethyl benzidine for HRP neurochemistry: a non-carcinogenic blue reaction-product with superior sensitivity for visualizing neural afferents and efferents. J Histochem Cytochem 1978; 26: 106-117.

Oades RD, Halliday GM. Ventral tegmental (A 10) system: neurobiology. I. Anatomy and connectivity. Brain Res Rev 1987; 12: 117-165.

Paxinos G, Watson Ch. The rat brain in stereotaxic coordinates. London, Sydney, Tokyo, Toronto: Academic Press, 1986.

Perciavalle V, Berretta S, Raffaele R. Projections from the intracerebellar nuclei to the ventral midbrain tegmentum in the rat. Neuroscience 1989; 29: 109-119.
Piazza PV, Ferdico M, Crescimanno G, Benigno A, Amato G. Inhibitory effect of the ventral tegmental A 10 region on the hypothalamic defence reaction: evidence for a possible dopaminergic mediation. Brain Res 1987; 413: 356-359.

Pollard JH. Handbook of Numerical and Statistical Techniques. Cambridge, London, New York, Melbourne: Cambridge University Press 1977.

Rossi F, Strata P. Reciprocal trophic interactions in the adult climbing fibre-Purkinje cell system. Prog Neurobiol 1995; 47: 341-369.

Shinoda Y, Futami T, Mitoma H, Yokota J. Morphology of single neurones in the cerebello-rubrospinal system. Behav Brain Res 1988; 28: 59-64.

Toyama K, Tsukahara N, Kosaka K, Matsunami K. Synaptic excitation of red nucleus by fibres from interpositus nucleus. Exp Brain Res 1970; 11: 187-198. 

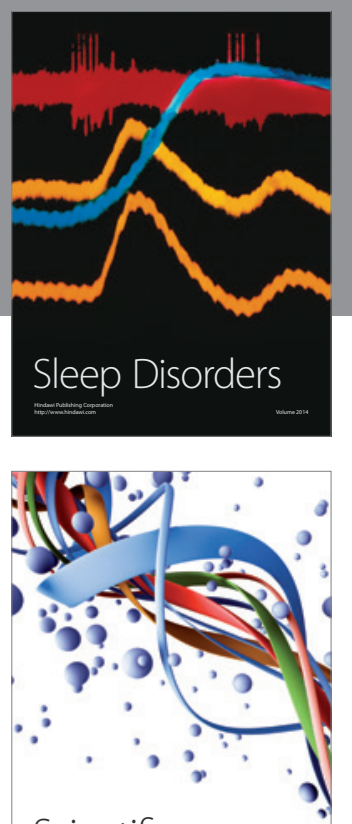

Scientifica
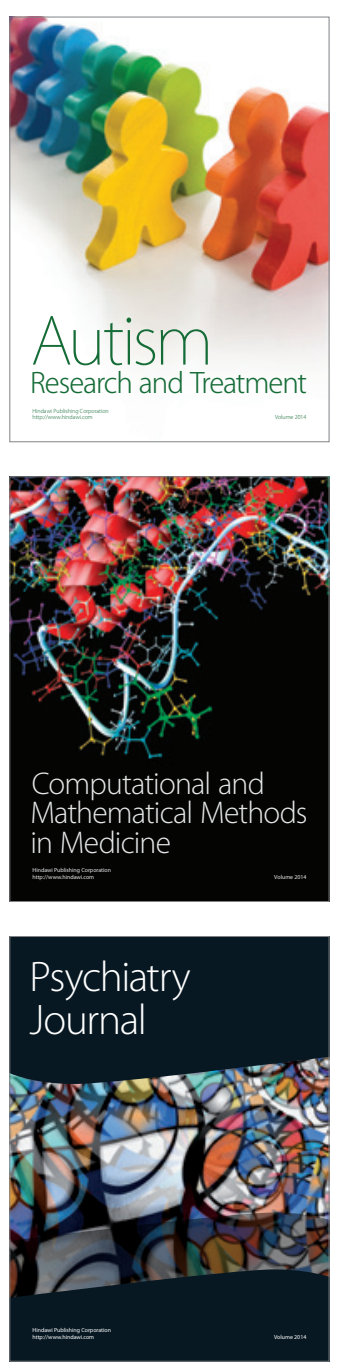
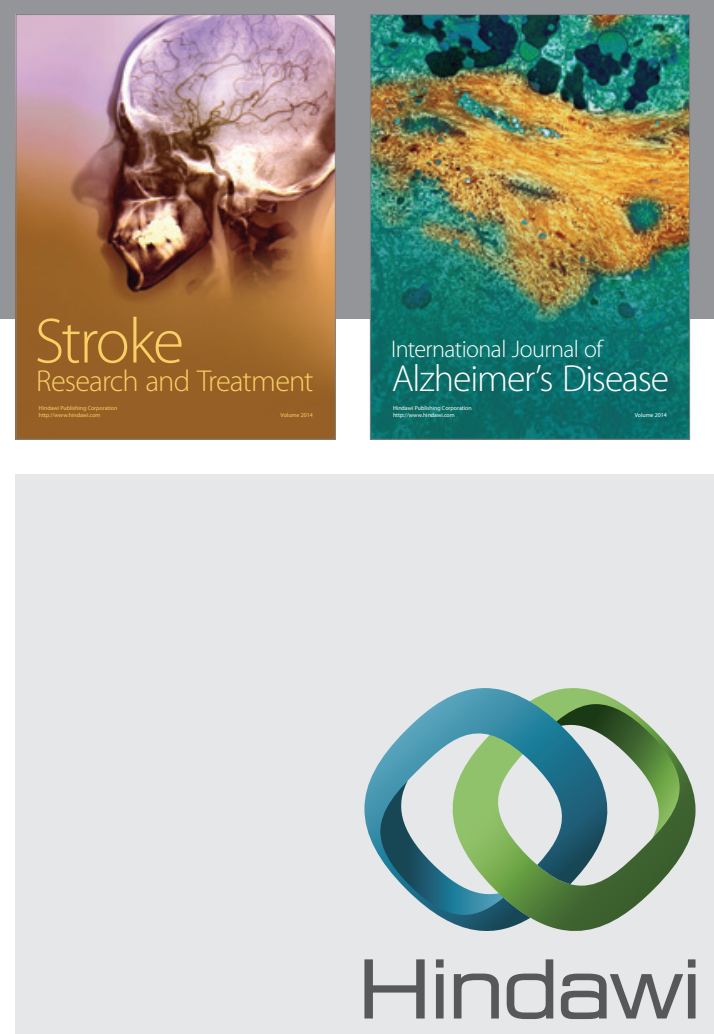

Submit your manuscripts at

http://www.hindawi.com
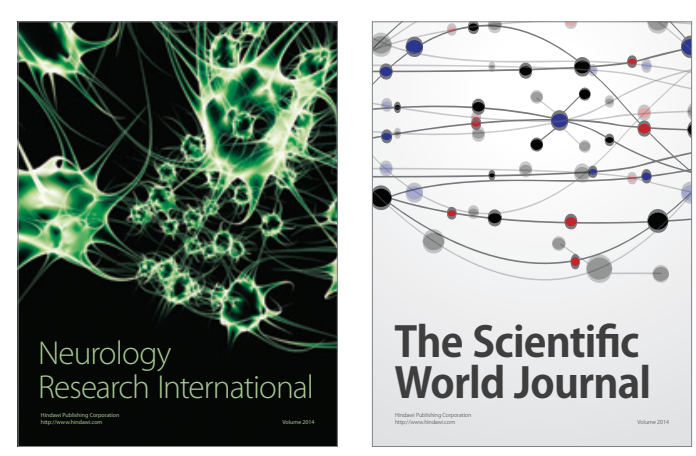

The Scientific World Journal

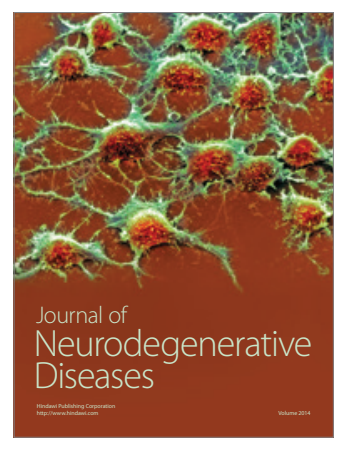

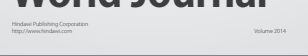

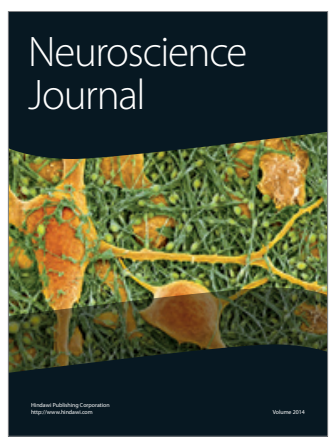

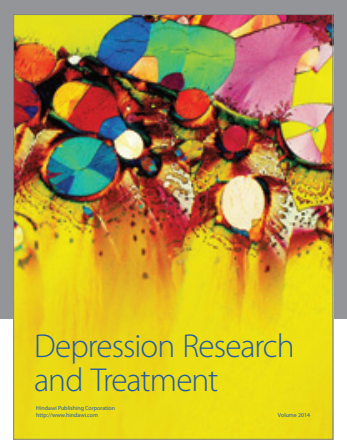
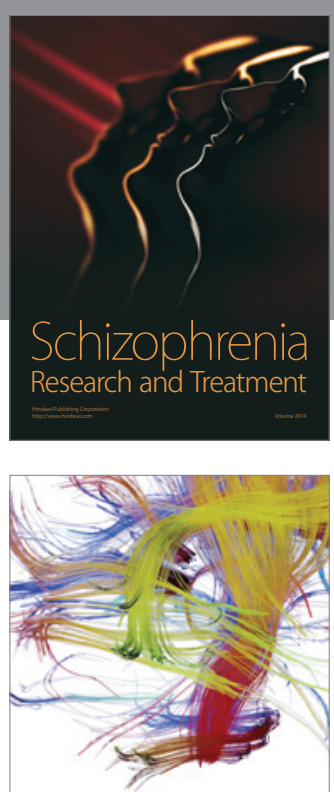

Brain Science

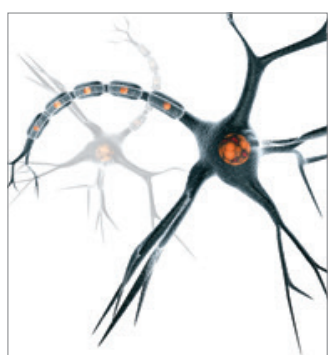

Neural Plasticity
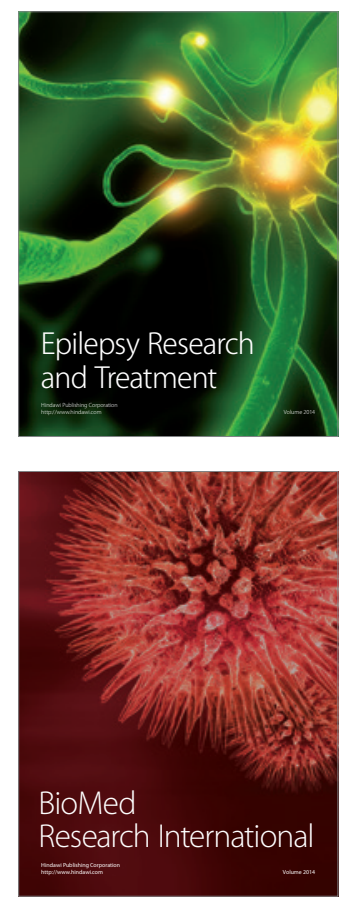

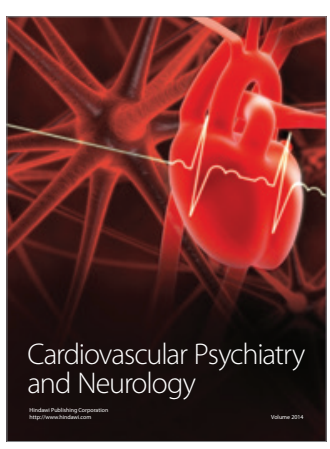

Parkinson's

Disease
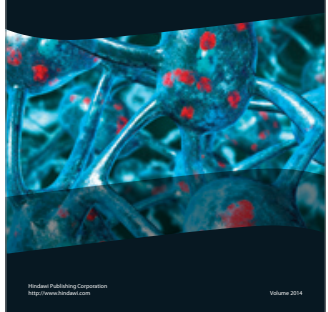